and in a prison environment. There was a combination of classroom learning and practice experience. An action plan was then formulated and implemented using Action Learning over a six month period.

Methods A five day programme (three days classroom based and two placement days). This was followed up with six action learning sets over the following six months. Each set was a two-hour period, where individuals' action learning was reviewed and summarised. Risk and obstacles were reflected upon and new action plans made if needed. The group was divided into two, each group having the same lead for the entire programme.

Results Individual and team objectives enabled the managers to lead in the change management of:

- Standardising daily board rounds

- Review of nursing handover processes

- The development of the role of a 'discharge coordinator'

- Improving access for BME patients

- Supporting staff to goal set with patients

- Undertake the leadership of key clinical skills - reviewing practice and policies e.g., tracheostomy care and Central venous access devices

- Reviewing processes with the HR department surrounding the management of sickness

- Setting an inpatient nursing action plan to support the hospice's strategic priories for 2017-2018.

Over the six months action learning was disrupted by staff leaving and shortages of staff over the summer holidays.

Conclusion All the managers evaluated the programme as a positive learning experience which enabled them to develop and lead practice changes.

\section{P-258 IVY ST!: A VIRTUAL CASELOAD FOR PALLIATIVE CARE EDUCATION}

Karen Groves. Queenscourt Hospice, Southport, UK

10.1136/bmjspcare-2018-hospiceabs.283

Background Palliative care education modules, developed collaboratively with the local university, required discussion about people living with progressive illness, their families and health professionals. It would not be possible to standardise real participant scenarios and the risks of lack of focus, loss of confidentiality and criticism high.

Aim To provide a virtual caseload/population with 'real' stories/situations moulded to participant needs.

Method The initial module had classroom sessions, supported by 'Blackboard' Virtual Learning Environment for participant access to resources. A web-based virtual community - 'Ivy St' with 'houses' (genograms) of extended families and nursing home. Each person had an avatar and a story, including a snapshot of their context - life, work, faith, hobbies and relationships. During the course a daily newsfeed of social and health activities on the street outlined clinical scenarios and role modelled the actions of health professionals with a continuously unfolding storyline.

Results Participants quickly engaged with the story and often expressed emotion about the happenings as if the characters were real. They seemed encouraged to discuss, pose possible conclusions from information available and suggest actions to be taken, learning from one another and online tutors. In the classroom, the topic being discussed would draw on street characters, with group-work on symptoms or treatments, conversations or services. Feedback from participants rated Ivy St as one of the course highlights, confirmed by a positive independent evaluation of the resource. Suggestions that too many happenings on the street in one day made it difficult to keep up with the story, changed the second iteration of the module.

Conclusion It was possible to replace discussion of real live cases, risking loss of confidentiality and criticism of actual care provision, with a virtual, standardisable, mouldable, web based community. The resource will be further developed for the second module and PGCert.

\section{P-259 THE EDUCATIONAL NEEDS OF PROFESSIONAL HOSPICE STAFF: AN ETHNOGRAPHIC INQUIRY}

\author{
${ }^{1,2}$ Andrea Mason. ${ }^{1}$ Alberta Health Senvices, Calgary, Canada; ${ }^{2}$ University of Calgary, \\ Calgary, Canada
}

\subsection{6/bmjspcare-2018-hospiceabs.284}

Problem Seniors are estimated at $25 \%$ of the Canadian population by the year 2036 (Alberta Health Services, 2014). Of those, 22\% will have a diagnosis of cancer yearly (Canadian Cancer Society's Advisory Committee on Cancer, 2015). In Alberta by the year 2030, there will be 27640 cases of cancer, with $25 \%$ requiring hospice care. These cancer cases comprise $85 \%$ of the hospice care in Alberta (Alberta Health Services, 2014). Several non-oncological diagnoses also require hospice care in Alberta (CSPCP, 2016). Inconsistent hospice staff knowledge has additionally become a concern for hospice care provincially (Alberta Health Services, 2014). No studies assessing the educational needs of Calgary hospice staff were found in a literature search. Also, international hospice nurses identified knowledge deficits in pain and symptom management, psychological, and spiritual care and communication with dying patients (Kehl, 2014; Ly Thuy, Yates, \& Osborne, 2014; Murray, Fiset \& O’Connor, 2004).

Purpose Determining ways hospice culture shapes the educational needs of professional staff. This knowledge provides information for staff education, promotes evidence-informed practice and improves hospice resident care.

Scope The study includes staff at a Calgary Catholic facility. This new site has been open for approximately two years at the time of the study. The hospice residents include those individuals with a life-limiting illness and a prognosis of approximately a few months.

Research design Qualitative, focused, interpretive ethnography. Data sources The data includes interviews with nine hospice staff members, the shadowing of those staff members, and field observations of the site culture focusing on staff educational needs. Data also includes; field notes, documents, and cultural artefacts.

Methodology The study design includes the researcher's interpretation of the hospice culture within the focused domain of staff educational needs. Data from the interviews, site observations and field notes are coded and analysed. This data reveals hospice cultural themes for analysis. 


\section{P-260 DEVELOPING AN ON-LINE TOOLKIT TO SUPPORT END OF LIFE CARE}

${ }^{1}$ Philippa Shreeve, ${ }^{2}$ Ruth England. ${ }^{1}$ Treetops Hospice Care, Derby, UK; ${ }^{2}$ Royal Derby Hospital, Derby, UK

\subsection{6/bmjspcare-2018-hospiceabs.285}

Background When the LCP was removed, a range of materials and guidance required review; furthermore, revised practice needed a platform in order to be shared. We recognised that a new approach might be supported by a novel on-line resource.

Methods A suite of tools that could be used by different providers across Derbyshire at different stages in a patient's end of life journey were collected together. Initially, these documents were held on a webpage hosted by a local community healthcare provider. An accompanying webpage contained details of upcoming educational events and contacts. In the first six months these pages had approximately 6500 hits, with each visit lasting on average $2.5 \mathrm{~min}$. However, feedback stated the pages were not easily accessible to all staff and were clumsy to use. Funding was obtained to build a more 'user friendly' resource. An improved platform was launched in October 2015.

Results In the period 1 October 2016 to 30 September 2017, 9862 sessions were conducted on our website by 5842 users. The average number of pages viewed per session was 3.6 with an average session duration being 3'34'. More than 50\% of users in this period were new to the website and our low bounce rate suggests that people who come to us find what they are looking for. The most popular resource is symptom management guidance. Most activity still comes from desktop computers but users do access the website via mobile devices. It is now possible to create an individualised learning portfolio using the toolkit. This can be linked to existing accredited resources (eELCA) as well as signposting to local face-to-face events.

Conclusions This novel website is a well-used platform for a suite of resources, as well as a means of educational support for those engaging in end of life care.

\section{P-261 ASSESSING THE IMPACT OF INTERVENTIONS TO PROMOTE CLINICAL EFFECTIVENESS IN A PALLIATIVE CARE SERVICE}

${ }^{1}$ Nicola Loveday, ${ }^{2}$ Matthew Curtis. ${ }^{1} T$ The Rowans Hospice, Waterlooville, UK; ${ }^{2}$ The Royal Bournemouth and Christchurch Hospitals NHS Foundation Trust, Bournemouth, UK

\subsection{6/bmjspcare-2018-hospiceabs.286}

Background Clinical effectiveness is a major component of clinical governance and integral to the successful operation of a palliative care service (PCS). Our PCS delivers regular internal educational programmes to share recent developments and latest knowledge and thereby promote high standards of care. Educational theory would suggest that different approaches might suit particular staff groups but it is not clear which approaches are best.

Aims To assess the most effective method to disseminate new learning throughout the PCS. To explore whether professional group is relevant to structure of learning event.

Method Delivery of the same material (highly relevant published paper, pertinent to specialists' practice) using two different approaches.
Session 1: CNS group. Paper distributed to all participants; read before the teaching session. Each allocated a section; presented findings at small group session. Researchers (two doctors) present to facilitate discussion.

Session 2: Doctors of all grades; researchers (same two doctors) presented the paper, reflecting on pertinent points. Structured questions throughout; highly interactive session.

Identical short survey sent before and after intervention to compare clinicians' baseline and post-teaching knowledge; a proxy measure of the effectiveness of said intervention.

Results - Both groups had improved knowledge postintervention.

- Baseline knowledge of CNSs was better than doctors (53\% v. $40 \%$ correct).

- CNSs demonstrated better post-teaching knowledge than doctors (95\% v. 78\%).

Conclusion This work highlights the importance of evaluating the impact of interventions to promote clinical effectiveness and seems to show that one form of intervention may be superior to the other. Future work should now focus on whether this 'more effective' approach is effective for doctors. Due to small numbers of learners, the team plan to continually assess these approaches over a number of topics. Further study to see whether this learning has a positive impact on practice and the outcomes for patients.

\section{P-262 TEN RECOMMENDATIONS FOR ORGANISATIONAL CHANGE TO BETTER SUPPORT CARERS AT END OF LIFE}

${ }^{1}$ Gail Ewing, ${ }^{2}$ Gunn Grande. 'University of Cambridge, Cambridge, UK; ${ }^{2}$ University of Manchester, Manchester, UK

\subsection{6/bmjspcare-2018-hospiceabs.287}

Background Carers play a vital role in supporting patients at end of life (EOL). EOL care policy promotes comprehensive person-centred assessment/support for carers, but without a clear implementation strategy this will only remain an aspiration.

Aim To develop recommendations for organisational structures/ processes required for implementation of comprehensive person-centred assessment/support for carers in EOL practice.

Methods 1) Secondary analysis of qualitative data on implementation in 36 services to identify factors facilitating/hindering implementation. 2) Expert consultation (focus groups with 19 lead practitioners/hospice managers) to review Stage1 findings and develop draft recommendations. 3) Wider stakeholder consultation - two professional workshops (23 participants: hospice, hospital, community, policy and academics), online survey (62 participants) and two carer workshops (nine participants) to finalise recommendations.

Results Comprehensive, person-centred carer assessment/support requires whole-systems change; a cultural shift at practitioner and organisational level. Ten recommendations identify key structures and processes not normally met by current provision:

- Consistent identification of carers within the care setting

- Demographic and contextual data on who the carer is and their situation

- A method/protocol for assessing carers and responding to assessment

- A recording system for carer information (separate from patient data) 